\title{
Improved model for atomic displacement calculation
}

\author{
Shengli Chen ${ }^{1,2, *}$, David Bernard ${ }^{1}$, Jean Tommasi ${ }^{1}$, and Cyrille De Saint Jean ${ }^{1}$ \\ ${ }^{1}$ CEA, Cadarache, DEN/DER/SPRC/LEPh, 13108 Saint Paul Les Durance, France \\ ${ }^{2}$ I-MEP2, University Grenoble Alpes, 38402 Saint Martin d'Heres, France
}

\begin{abstract}
Atomic displacement is one of the key factors that influence the behaviors of material properties during and after irradiation. Many models, including the international standard metric Norgett-Robinson-Torrens model (NRT), have been developed to calculate the number of Displacement per Atom (DPA) using the energy of Primary Knocked-on Atom (PKA) as a major parameter. However, extensive experiments and simulations indicate that the NRT-DPA model seriously overestimates (about 3 times) the actual DPA. Nordlund recently developed the Athermal Recombination-Corrected DPA (ARC-DPA) model, which shows that the Molecular Dynamics (MD) simulations can be directly used to compute DPA by fitting the simulated data for each isotope. The present work proposes a simpler expression for the efficiency function to calculate the DPA without requiring fitting parameters as needed in the ARC-DPA model. Our DPA calculation results utilizing the improved efficiency function are validated against the experimental data for the $\mathrm{Fe}, \mathrm{Ni}, \mathrm{Cu}$, and $\mathrm{Ag}$. The applications in fast breeder nuclear reactors show good agreement with the ARC-DPA metric for ${ }^{56} \mathrm{Fe}$.
\end{abstract}

\section{Introduction}

In the nuclear industry, the operating lifetime of a Light Water Reactor (LWR) is determined by the life of Reactor Pressure Vessel (RPV), which contains the reactor core and is irradiated by neutrons and photons (and other particles) produced by nuclear reactions. The accurate knowledge about the irradiation damage, which is conventional quantified by the number of Displacement per Atom (DPA), of RPV can help to decrease the safety margin for the life length of a LWR. In order to compute the DPA rate in materials, many methods and empirical formulae have been proposed by using the Primary Knock-on Atom (PKA) kinetic energy as a major parameter.

Based on extensive Binary Collision Approximation (BCA) [1] simulations, Norgett, Robinson, and Torrens (NRT) proposed the modified Kinchin-Pease [2] formula in 1975 [3]. The NRT formula gives the number of DPA:

$$
N\left(E_{a}\right)= \begin{cases}0 & E_{a}<E_{d} \\ 1 & E_{d}<E_{a}<2 E_{d} / 0.8 \\ 0.8 E_{a} / 2 E_{d} & E_{a}>2 E_{d} / 0.8\end{cases}
$$

where $E_{a}$ is energy available to create displacement of atoms by collisions, referred to damage energy [4], $E_{d}$ is the average threshold energy of atomic displacement [5], and 0.8 is the displacement efficiency obtained by the BCA simulations (the original value given in Ref. [1] is 0.86). The damage energy is calculated by $E_{a}=E_{P K A} \times f\left(E_{P K A}\right)$, where $f$ is the partition function which describes the fraction of $E_{P K A}$ left in atomic motion [4]. The NRT-DPA formula is nowadays used as the international standard of primary radiation damage.

*e-mail: shengli.chen@cea.fr
However, the overestimation of DPA in the NRT metric was found by comparison with experimental data for copper $(\mathrm{Cu})$ and silver $(\mathrm{Ag})$ [6]. Improved models for predicting damage cross sections and associated uncertainties are thus of importance. One of the issues in the NRT model is that the in-cascade recombination of displaced atoms is not considered. Taking this effect into account, the Athermal Recombination-Corrected-DPA (ARC-DPA) is proposed by Nordlund et al. [7]. The relative damage efficiency $\xi$ is defined as the ratio of the "true" number of Frenkel Pairs (FP) to the number of FP calculated with NRT formula. Its expression is based on the fact that the number of FP $\left(N_{F P}\right)$ tends to $a^{\prime} E_{a}$ when $E_{a}$ tends to infinity and $N_{F P}$ tends to $c^{\prime} E_{a}^{0.8}$ at low energy. Therefore, the following simple function is proposed:

$$
N_{F P}=a^{\prime} E_{a}^{b+1}+c^{\prime} E_{a}
$$

Accordingly, the efficiency becomes:

$$
\xi\left(E_{a}\right)=N_{F P} / N_{N R T}=a E_{a}^{b}+c
$$

By continuity of DPA, one impose an additional condition $\xi\left(2 E_{d} / 0.8\right)=1$ so that:

$$
\xi\left(E_{a}\right)=\left(1-c_{\text {arc }}\right) \times\left[0.8 E_{a} / 2 E_{d}\right]^{b_{\text {arc }}}+c_{\text {arc }}
$$

The coefficients $b_{\text {arc }}$ and $c_{\text {arc }}$ are fitted through the results of Molecular Dynamics (MD) simulations. The values of $b_{\text {arc }}$ and $c_{\text {arc }}$ for $\mathrm{Fe}, \mathrm{Ni}$, and $\mathrm{Cu}$ are listed in Table 1 . Within this adjustment, the effective and empiric ARC-DPA formula is given by:

$$
N\left(E_{a}\right)= \begin{cases}0 & E_{a}<E_{d} \\ 1 & E_{d}<E_{a}<2 E_{d} / 0.8 \\ 0.8 E_{a} / 2 E_{d} \xi\left(E_{a}\right) & E_{a}>2 E_{d} / 0.8\end{cases}
$$


Due to the utilization of MD simulations, the ARCDPA can generally much better describe the DPA than the NRT formula. However, one of the drawbacks of the ARC model is that MD simulation results are required for each isotope. Therefore, the present work proposes a simple efficiency function without introducing additonal parameters compared with the NRT-DPA metric.

\section{Method}

The differential equation of annihilation is:

$$
d n / d t=-k n^{2}
$$

where $n$ represents the concentration of point defects of either kind. By integrating over the time, the solution of Eq. 6 can be calculated:

$$
n(t)=\frac{n(t=0)}{1+k n(t=0) t}
$$

Inspiring from the equation of annihilation, Robinson and Torrens proposed a function of average energy dissipated to each displaced atom (analogue to $n(t=0) / n(t)$ ) as [1]:

$$
E_{a} / N_{F P}=2 E_{d} / 0.8+\alpha E_{a}
$$

where $\alpha$ is a fitted coefficient. The efficiency function based on the NRT-DPA formula is defined as:

$$
\xi\left(E_{a}\right)=N_{F P} / N R T=N_{F P} /\left(0.8 E_{a} / 2 E_{d}\right)
$$

Inserting Eq. 8 to Eq. 9:

$$
\xi\left(E_{a}\right)=\frac{2 E_{d} / 0.8}{2 E_{d} / 0.8+\alpha E_{a}}
$$

The above efficiency function tends to 0 by increasing the damage energy. However, the defect production is a linear function of the damage energy after the formation of displacement sub-cascade at high energy collisions $[8,9]$. The efficiency should thus be a constant after the subthreshold energy, which defines the threshold of subcascade formation. Both MD simulations and experimental data show the about $1 / 3$ asymptotic value of the efficiency for many isotopes. Consequently, the asymptotic value of the efficiency $(\beta)$ should be taken into account:

$$
\xi\left(E_{a}\right)=\frac{2 E_{d} / 0.8}{2 E_{d} / 0.8+\beta E_{a}}+\beta
$$

where $\beta=Z /(1.5 A)$ [in which $Z$ is the atomic number and $A$ is the atomic mass] is considered as the asymptotic value of $\xi\left(E_{a}\right) . \beta \propto Z / A$ because stronger Coulomb force or lighter mass leads to longer path that induces "more" sub-cascades. The advantage of this improved efficiency function is that we correlate the atomic displacement efficiency with the atomic number and the atomic mass of the irradiated material without requiring fitting parameters as needed in the ARC-DPA model. In addition, the same $\beta$ is used for the coefficient in the denominator of the first term to simplify the expression. This improved correction is hereinafter referred to Chen-Bernard (CB).
Table 1. Parameters for DPA calculation

\begin{tabular}{lllll}
\hline Element & $E_{d}(\mathrm{eV})$ & $b_{\text {arc }}$ & $c_{\text {arc }}$ & Ref. \\
\hline $\mathrm{Fe}$ & 40 & -0.568 & 0.286 & {$[7]$} \\
\hline $\mathrm{Ni}$ & 39 & -1.01 & 0.23 & {$[7]$} \\
\hline $\mathrm{Cu}$ & 33 & -0.68 & 0.16 & {$[7]$} \\
\hline $\mathrm{Ag}$ & 39 & -1.06 & 0.257 & {$[15]$} \\
\hline
\end{tabular}
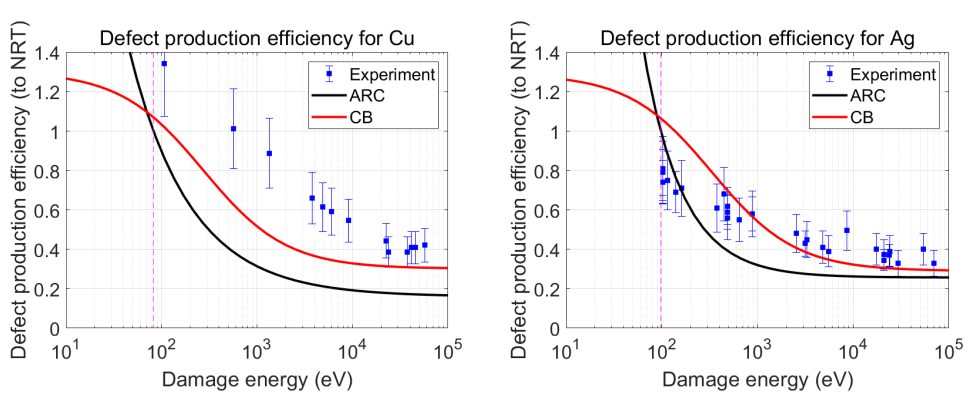

Figure 2. Ratios of Averback's experimental data [6], ARCDPA, and CB-DPA to the NRT formula for $\mathrm{Cu}$ and $\mathrm{Ag}$ versus damage energy computed with the DPA weighted average PKA energy. The corresponding uncertainties are form the uncertainties of sample thickness and electronic resistivity.

\section{Results and discussion}

\subsection{Validation of the current DPA formula}

The ratios of ARC and CB to the NRT metric for the Fe, $\mathrm{Ni}$, and $\mathrm{Cu}$ are shown in Figure 1. Fe and $\mathrm{Ni}$ are two most important elements in the RPV for radiation damage investigations. All the three elements are of importance in fusion reactors. The values of $b_{a r c}$ and $c_{a r c}$ in ARC-DPA formula and the threshold energy $E_{d}$ for these elements are compiled by Nordlund [7] and listed in Table 1. The original results of MD simulations can be found in Ref. [10] and Ref. [11] for Fe and Ni, respectively. The experimental data are extracted from the Nuclear Energy Agency (NEA) report [12], which accounts for the experimental measurements of Jung [13]. The DPA is proportional to the Resistivity of Frenkel pairs per unit concentration (RF) (c.f. Eq. (1) in Ref. [6]). The experimental uncertainties are deduced from the RF values compiled in Tables 5 and 16 in Ref. [13]. It can be found that the CB corresponds well with the experimental data. The discrepancies between the CB model and the experimental values are even less than the ARC-DPA model for $\mathrm{Fe}, \mathrm{Ni}$, and $\mathrm{Cu}$.

Averback deduced experimental DPA numbers of copper and silver according to the measurement of electrical resistivity [6]. The data of Averback are given as a function of DPA averaged PKA energy, which is larger than the average PKA energy. However, these data can reveal the asymptotic values of displacement efficiency. The total uncertainty of Averback's DPA comes from the uncertainties of the thickness of samples (5\% for samples No. $2 \& 3$ and $10 \%$ for other samples) and the correction to convert measured thin film resistivities (10\%). The ratios of ARC and CB to the NRT metric for the $\mathrm{Cu}$ and $\mathrm{Ag}$ are shown in Figure 2 with Averback's experimental data. The experimental to NRT ratios for $\mathrm{Cu}$ is corrected 

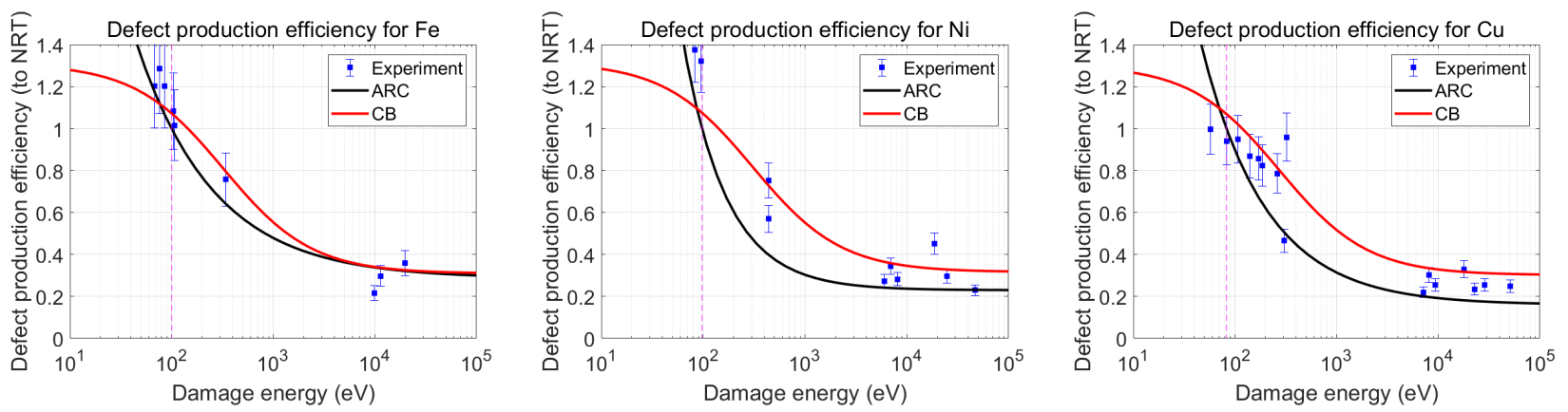

Figure 1. Ratios of experimental data, ARC-DPA, and CB-DPA to the NRT formula for $\mathrm{Fe}, \mathrm{Ni}$, and $\mathrm{Cu}$ versus damage energy. The dashed pink lines indicate $2.5 E_{d}$. The experimental data are extracted from Nuclear Energy Agency (NEA) report [12], which accounts for the experimental measurements of Jung [13]. The experimental uncertainties are deduced from Tables 5 and 16 in Ref. [13] through the Resistivity of Frenkel pairs per unit concentration, to which the number of DPA is proportional [6].

because Averback adopted Lucasson's threshold energy 29 $\mathrm{eV}$ [14]. The ARC-DPA parameters of Ag are listed in the last row in Table 1 [15].

Figure 1 shows that the $\mathrm{CB}$ formula corresponds well to experimental data for $\mathrm{Fe}, \mathrm{Ni}$, and $\mathrm{Cu}$. Results in Figure 2 show the good agreement of the asymptotic value of the CB-DPA for Ag. The asymptotic value of Averback's experimental measurement is higher than both ARC-DPA and CB-DPA, while the experimental data shown in Figure 1 are between ARC-DPA and CB-DPA. Comparing with the NRT metric, no additional parameters are used in the CB formula. The simpler form of the CB than that of the ARC results in quicker calculations for the propagation of uncertainties from nuclear parameters to DPA rates. In addition, $\mathrm{MD}$ simulations are required for each isotope to fit the parameters $b_{\text {arc }}$ and $c_{\text {arc }}$ in the ARC-DPA metric. Konobeyev et al. tried to find a systematics to determine the parameter $c_{\text {arc }}$ in ARC-DPA, but the discrepancies between the systematics and the MD simulations are still evident [15]. Therefore, the CB-DPA formula can be used to approximatively compute the atomic displacement damage if no MD simulation result is available. It is noteworthy that the $c_{\text {arc }}$ in ARC-DPA formula is not necessarily close to $1 / 3$.

\subsection{Examination in fast neutron reactors}

Since the DPA is one of the key factors in nuclear reactor studies and the major DPA in a reactor is induced by neutrons, the calculations of neutron induced DPA rates are performed on two experimental fast breeder reactors in the present work. For an external particle induced atomic displacements in materials, the mechanism is that the PKA obtains energy from the collision with the incident particle. It requires thus the calculation of the recoil energy of the PKA to determine the DPA for an external particle induced displacement [16].

The DPA rate induced by a type of external particle is calculated by [17]:

$$
D P A=\frac{0.8}{2 E_{d}} \int_{0}^{\infty} \Sigma_{i}\left[E_{a, i}(E) \xi\left(E_{a, i}\right) \sigma_{i}(E)\right] \phi(E) d E
$$

where the index $i$ reveals the reaction type, $\sigma_{i}(E)$ is the cross section of the reaction $i$ at energy $E, \xi\left(E_{a, i}\right)$ is the efficiency of displacement based on the NRT metric, it is equal to unity for the NRT formula, Eq. 4 and Eq. 11 are used for the ARC-DPA formula and CB-DPA formula, respectively, $\phi(E)$ refers to the spectrum of the incident particle.

The quantity in bracket in Eq. 12 is defined as the DPA cross section in barn.eV. The ARC model for the Fe isotopes and the CB model for all isotopes are implemented in the processing code NJOY2016 [18] in this study. The numeric verification of DPA cross sections is presented in Ref. [19]. The DPA cross sections of ${ }^{56} \mathrm{Fe}$ calculated with the lastest released JEFF-3.3 library [20] is presented in Figure 3 for the NRT, ARC, and CB formulae, respectively. Small difference between the ARC-DPA and CBDPA cross sections is observed. The Stainless Steel (SS) is used for the material of the reactor pressure vessel in LWR and fuel cladding in fast neutron reactors. Recent study reveals the candidature of the SS FeCrAl in the fuel cladding of LWR [21, 22]. The main constitution in the stainless steel is the iron, and ${ }^{56} \mathrm{Fe}$ constitutes $91.75 \%$ natural iron element. The DPA calculations of ${ }^{56} \mathrm{Fe}$ are thus performed.

The neutronic simulations of the European Fast Reactor (EFR) [23] is performed with ERANOS [24]. Results show that $27 \mathrm{dpa} /$ year of ${ }^{56} \mathrm{Fe}$ in fuel cladding is obtained when the NRT model is used. The ARC-DPA and the CBDPA models show $9.2 \mathrm{dpa} /$ year and $9.6 \mathrm{dpa} /$ year, respectively. The ratios of DPA rates calculated with different models to that calculated with the NRT metric are given in Table 2. The last row in Table 2 shows the same quantities but in the cladding of Phenix fast nuclear reactor, of which the neutron spectrum is calculated by using the JEFF-3.1.1 nuclear data library [25] and the $P_{i j}$ deterministic neutron transport method in APOLLO-2 [26].

Both Figure 1 and Figure 3 show the small difference between CB-DPA and ARC-DPA for ${ }^{56} \mathrm{Fe}$. Results in Table 2 confirm that the ARC and the CB models have similar results on ${ }^{56} \mathrm{Fe}$ DPA calculations in fast neutron reactors, while the $\mathrm{CB}$ formula has a simpler form and does 


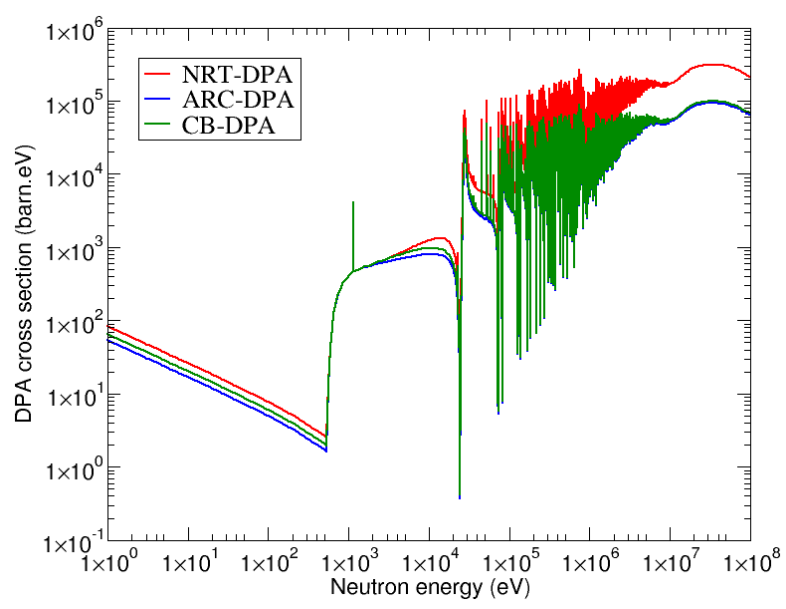

Figure 3. DPA cross sections of ${ }^{56} \mathrm{Fe}$ based on the JEFF-3.3

Table 2. Ratios of DPA calculated with different models to that calculated with the NRT metric for ${ }^{56} \mathrm{Fe}$ in the fuel cladding of different reactors

\begin{tabular}{llll}
\hline Reactor & NRT & ARC-DPA & CB-DPA \\
\hline EFR & 1.00 & 0.342 & 0.358 \\
Phenix & 1.00 & 0.339 & 0.354 \\
\hline
\end{tabular}

not require experimental or simulations data to determine the parameters.

\section{Conclusions}

The recently developed ARC-DPA model computes DPA with MD simulations results. The MD-based library of DPA calculations is possible thanks to the ARC-DPA. Inspiring from the annihilation equation, we propose an efficiency function with a simpler form without introducing additional parameters excluded in the current international standard NRT-DPA formula. The simpler form of CB-DPA can simplify the uncertainty propagation, while the calculations of sensitivities to fitted coefficients are required for ARC-DPA. The CB-DPA is validated against with experimental data for $\mathrm{Fe}, \mathrm{Ni}, \mathrm{Cu}$, and $\mathrm{Ag}$. In addition, the applications in fast breeder nuclear reactors show good agreement with the ARC-DPA metric for ${ }^{56} \mathrm{Fe}$. Therefore, the CB-DPA formula can be used to approximatively compute the DPA rates in the case of no MD data is available.

\section{References}

[1] M. T. Robinson and I. M. Torrens, Phys. Rev. B 9(12), 5008-5024 (1974)

[2] G. H. Kinchin and R. S. Pease, Rep. Prog. Phys. 18(1), 1-51 (1955)
[3] M. J. Norgett, M. T. Robinson, and I. M. Torrens, Nucl. Eng. Des. 33(1), 50-54 (1975)

[4] J. Lindhard, V. Nielsen, M. Scharff, and P. V. Thomsen, Mat. Fys. Medd. Dan. Vid. Selsk. 33(10), 1-42 (1963)

[5] C. H. M. Broeders and A. Yu. Konobeyev, J. Nucl. Mater. 328(2), 197-214 (2004)

[6] R. S. Averback, R. Benedek, and K. L. Merkle, Phys. Rev. B, 18(8), 4156 (1978)

[7] K. Nordlund et al., Nat. Commun., 9(1), (2018).

[8] K. Nordlund, M. Ghaly, R. S. Averback, M. Caturla, T. Diaz de la Rubia, and J. Tarus, Phys. Rev. B, 57(13), 7556-7570 (1998)

[9] J.-P. Crocombette, L. Van Brutzel, D. Simeone, and L. Luneville, J. Nucl. Mater., 474, 134-142 (2016)

[10] C. Bjorkas and K. Nordlund, Nucl. Instrum. Methods Phys. Res. Sect. B Beam Interact. Mater. At., 259(2), 853-860 (2007)

[11] A. E. Sand and K. Nordlund, J. Nucl. Mater., 456, 99-105 (2015)

[12] K. Nordlund et al., NEA/NSC/DOC(2015)9, (2015)

[13] P. Jung, Atomic Defects in Metals 25, (SpringerVerlag, Berlin/Heidelberg, 1991)

[14] P. Lucasson, Fundamental aspects of radiation damage in metals 1, (ORNL, US, 1975) 42-65

[15] A. Yu. Konobeyev, U. Fischer, Yu. A. Korovin, and S. P. Simakov, Nucl. Energy Technol., 3(3), 169-175 (2017)

[16] S. Chen and D. Bernard, J. Nucl. Mater., 522, 236245 (2019)

[17] S. Chen, D. Bernard, and L. Buiron, Nucl. Eng. Des., 346, 85-96 (2019)

[18] R. E. MacFarlane, D. W. Muir, R. M. Boicourt, A. C. Kahler, and J. L. Conlin, LANL, LA-UR-17-20093 (2016)

[19] S. Chen et al.,, Nucl. Instrum. Methods Phys. Res. Sect. B, 456, 120-132 (2019)

[20] NEA, JEFF-3.3 Nuclear Data library (2017)

[21] S. Chen and C. Yuan, Sci. Technol. Nucl. Install., 2017, 3146985 (2017)

[22] S. Chen, C. Yuan, and D. Guo, Ann. Nucl. Energy, 124, 460-471 (2019)

[23] J. C. Lefevre, C. H. Mitchell, and G. Hubert, Nucl. Eng. Des., 162(2-3), 133-143 (1996)

[24] J. M. Ruggieri et al., Proceedings of ICAPP 06, Reno, NV USA, 2432-2439 (2006)

[25] A. Santamarina et al., JEFF Report 22, NEA No. 6807, (2009)

[26] A. Santamarina et al., Advances in Nuclear Fuel Management IV (ANFM 2009), Hilton Head Island, South Carolina, USA, 1-18 (2009) 